\title{
The Primordial Inflation Explorer (PIXIE) Mission
}

\author{
Alan J. Kogut ${ }^{\mathrm{a}}$, David T. Chuss ${ }^{\mathrm{a}}$, Jessie L. Dotson ${ }^{\mathrm{b}}$, Dale J. Fixsen ${ }^{\mathrm{a}}$, Mark Halpern ${ }^{\mathrm{c}}$, \\ Gary F. Hinshaw ${ }^{a}$, Stephan M. Meyer ${ }^{d}$, S. Harvey Moseley ${ }^{a}$, Michael D. Seiffert ${ }^{d}$, \\ David N. Spergel ${ }^{\mathrm{f}}$, Edward J. Wollack ${ }^{\mathrm{a}}$ \\ ${ }^{a}$ Code 665, NASA Goddard Space Flight Center, Greenbelt, MD, USA 20771; \\ NASA Ames Research Center, Moffett Field, CA, USA 94035; \\ ${ }^{c}$ Dept. of Physics \& Astronomy, University of British Columbia, Vancouver, BC, Canada, V6T 1Z1; \\ ${ }^{\mathrm{d} D e p t}$ of Astronomy \& Astrophysics, University of Chicago, Chicago, IL, USA; \\ ${ }^{\mathrm{e}} \mathrm{Jet}$ Propulsion Laboratory, MS 169-506, Pasadena, CA, USA 91109; \\ fDept of Astrophysical Sciences, Princeton University, Princeton, NJ, USA 08544
}

\begin{abstract}
The Primordial Inflation Explorer (PIXIE) is an Explorer-class mission to map the absolute intensity and linear polarization of the cosmic microwave background and diffuse astrophysical foregrounds over the full sky from frequencies $30 \mathrm{GHz}$ to $6 \mathrm{THz}(1 \mathrm{~cm}$ to $50 \mu \mathrm{m}$ wavelength). PIXIE uses a polarizing Michelson interferometer with $2.7 \mathrm{~K}$ optics to measure the difference spectrum between two orthogonal linear polarizations from two co-aligned beams. Either input can view either the sky or a temperature-controlled absolute reference blackbody calibrator. The multimoded optics and high etendu provide sensitivity comparable to kilo-pixel focal plane arrays, but with greatly expanded frequency coverage while using only 4 detectors total. PIXIE builds on the highly successful COBE/FIRAS design by adding large-area polarization-sensitive detectors whose fully symmetric optics are maintained in thermal equilibrium with the CMB. The highly symmetric nulled design provides redundant rejection of major sources of systematic uncertainty. The principal science goal is the detection and characterization of linear polarization from an inflationary epoch in the early universe, with tensor-to-scalar ratio $r \ll 10^{-3}$. PIXIE will also return a rich data set constraining physical processes ranging from Big Bang cosmology, reionization, and large-scale structure to the local interstellar medium.
\end{abstract}

Keywords: cosmic microwave background, polarization, FTS, bolometer

\section{INTRODUCTION}

A central principle in modern cosmology is the concept of inflation, which posits a period of exponential expansion in the early universe shortly after the Big Bang. The many e-foldings of the scale size during inflation force the geometry of space-time to asymptotic flatness while dilating quantum fluctuations in the inflaton potential to the macroscopic scales responsible for seeding large-scale structure in the universe. Inflation provides a simple, elegant solution to multiple problems in cosmology, but it relies on extrapolation of physics to energies more than 12 orders of magnitude beyond those accessible to particle accelerators.

Measurement of the linear polarization of the cosmic microwave background (CMB) provides a direct test of inflationary physics. CMB polarization results from Thomson scattering of CMB photons by free electrons. Scattering of an isotropic radiation field produces no net polarization, but a quadrupole moment in the incident radiation yields a polarized signal. The required quadrupole can result from either temperature anisotropy in the radiation field itself, or the differential redshift as gravity waves propagate through an isotropic medium. Temperature or density perturbations are scalar quantities; their polarization signal must therefore be curl-free. Gravity waves, however, are tensor perturbations whose polarization includes both gradient and curl components. In analogy to electromagnetism, the scalar and curl components are often called " $E$ " and " $B$ " modes. Only gravity waves induce a curl component: detection of a B-mode signal in the CMB polarization field is recognized as a "smoking gun" signature of inflation, testing physics at energies inaccessible through any other means ${ }^{1,2,3}$.

*Alan.J.Kogut@nasa.gov; phone 1301-286-0853 


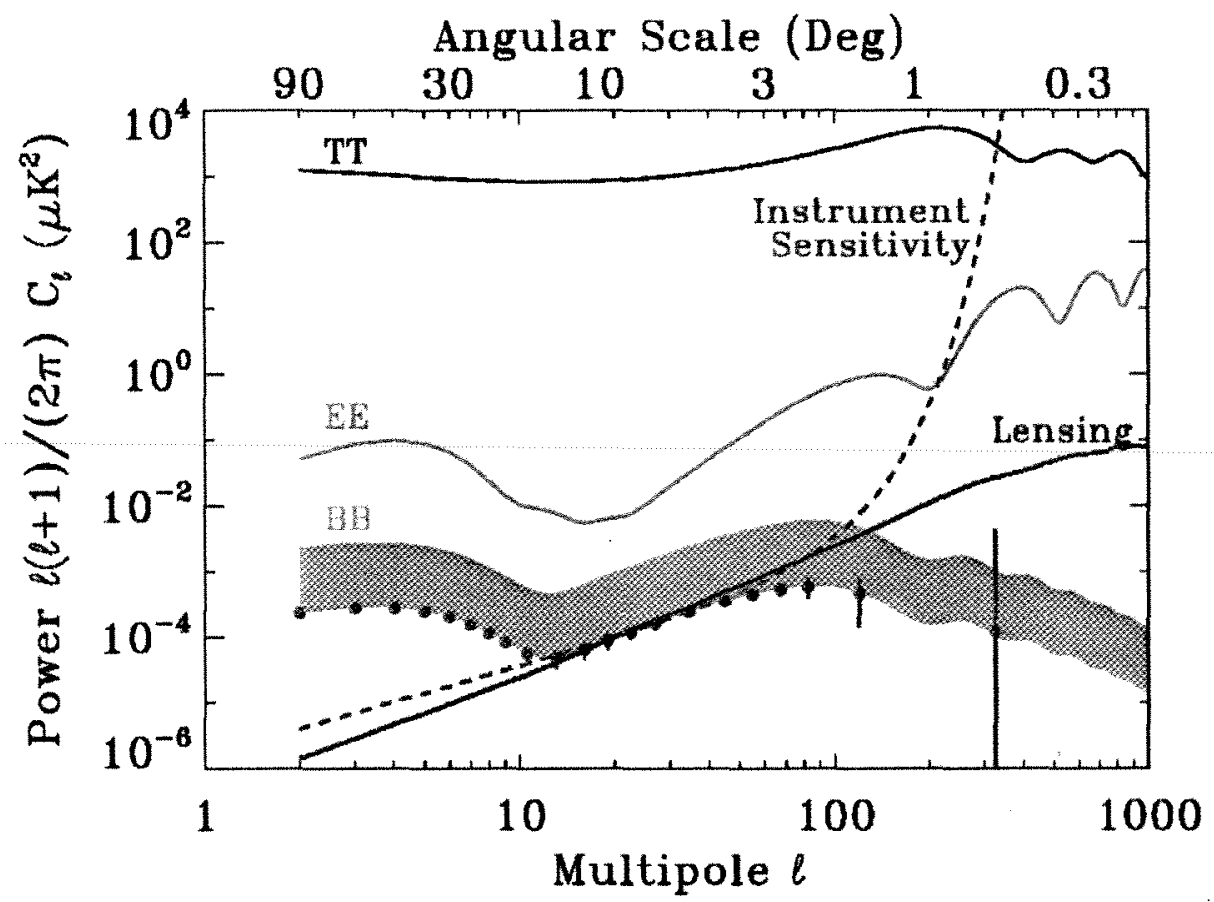

Figure 1. Angular power spectra for unpolarized (TT), E-mode (EE), and B-mode (BB) polarization. The blue band shows the predicted range for the inflationary signal. The PIXIE un-binned sensitivity (dashed red line) matches the confusion noise from gravitational lensing of the primordial E-mode signal. PIXIE can measure even the minimum predicted B-mode power spectrum at high statistical confidence (red points and error bars).

Figure 1 shows the predicted angular power spectra of the E- and B-mode polarization. The simplest inflationary models predict an observable B-mode signal, with amplitude in the range 30--100 $\mathrm{nK}$ thermodynamic temperature (blue band). Detection of this signal would have profound consequences. The amplitude $r$ of the gravity-wave signal depends directly upon the energy scale of inflation,

$$
V^{1 / 4}=1.06 \times 10^{16} \mathrm{GeV}\left(\frac{r}{0.01}\right)^{1 / 4}
$$

where $r$ is conventionally expressed as the ratio of the tensor to scalar power. A positive detection would not only establish inflation as a physical reality, but would provide a model-independent determination of the relevant energy scale to test physics at energies near Grand Unification. Characterization of the B-mode power spectrum would additionally probe the trans-Planckian physics between the beginning and end of the inflationary epoch, providing direct observational input to the ultraviolet completion of quantum field theory and gravity ${ }^{4}$.

Detecting the inflationary signal is difficult. As recognized in a series of reports ${ }^{5.6}$, there are three fundamental challenges: sensitivity, foregrounds, and systematic errors.

- Sensitivity: The inflationary signal is faint compared to the fundamental sensitivity limit imposed by photon statistics.

Even noiseless detectors suffer from this photon-counting limit; the only solution is to collect more photons. Conventional designs accomplish this using large (kilo-pixel) detector arrays, adversely impacting both mission complexity and cost.

- Foregrounds: A second challenge for CMB polarimetry is separating the CMB signal from Galactic foregrounds. Foreground emission within the Galaxy is polarized and is brighter than the dominant E-mode polarization even at the foreground minimum near $60 \mathrm{GHz}$ (Figure 2). Detecting the much fainter B-mode signal requires foreground cleaning effective at the few percent level, which in turn requires multiple frequency channels to measure the amplitude, polarization vector, and frequency dependence of each foreground component. 
- Systematic Errors: The gravity-wave signal is less than $1 \%$ of E-mode polarization, which in turn is only a few percent of the unpolarized temperature fluctuations on the sky. Even a small admixture of power from either unpolarized sources or the dominant E-mode polarization into a spurious B-mode pattern could overwhelm the primordial signal. The mission design must rigorously control stray light and the instrumental separation of polarized from unpolarized signals in order to confidently detect primordial polarization.

Satisfying the simultaneous requirements of sensitivity, foreground discrimination, and immunity to systematic errors presents a technological challenge. Most instrument designs currently fielded combine focal-plane arrays containing hundreds to thousands of detectors arranged in a modest number (typically 3 to 6) of frequency channels. In this paper, we describe an instrument capable of measuring the CMB and diffuse foregrounds with background-limited sensitivity in over 500 frequency channels using only 4 detectors. The resulting reduction in complexity and cost make the instrument an attractive candidate for a future Explorer mission.

\section{INSTRUMENT DESCRIPTION}

The Primordial Inflation Explorer (PIXIE) is a mission concept designed to detect the polarization signal from an inflationary epoch while remaining within the constraints of NASA's Explorer program. It consists of a polarizing Michelson interferometer which interferes two optical inputs to measure the fringe pattern as a function of the optical phase delay. It uses only 4 semiconductor bolometers to measure both the frequency spectrum and polarization state of the sky to $\mathrm{nK}$ precision at wavelengths $1 \mathrm{~cm}$ to $50 \mu \mathrm{m}$. Observing from low Earth orbit, it can achieve B-mode sensitivity $\mathrm{r} \ll 10^{-3}$ while also providing important new results ranging from the ionization history of the early universe to the spectrum and anisotropy of the far-infrared background to the chemical and energy balance of the interstellar medium.

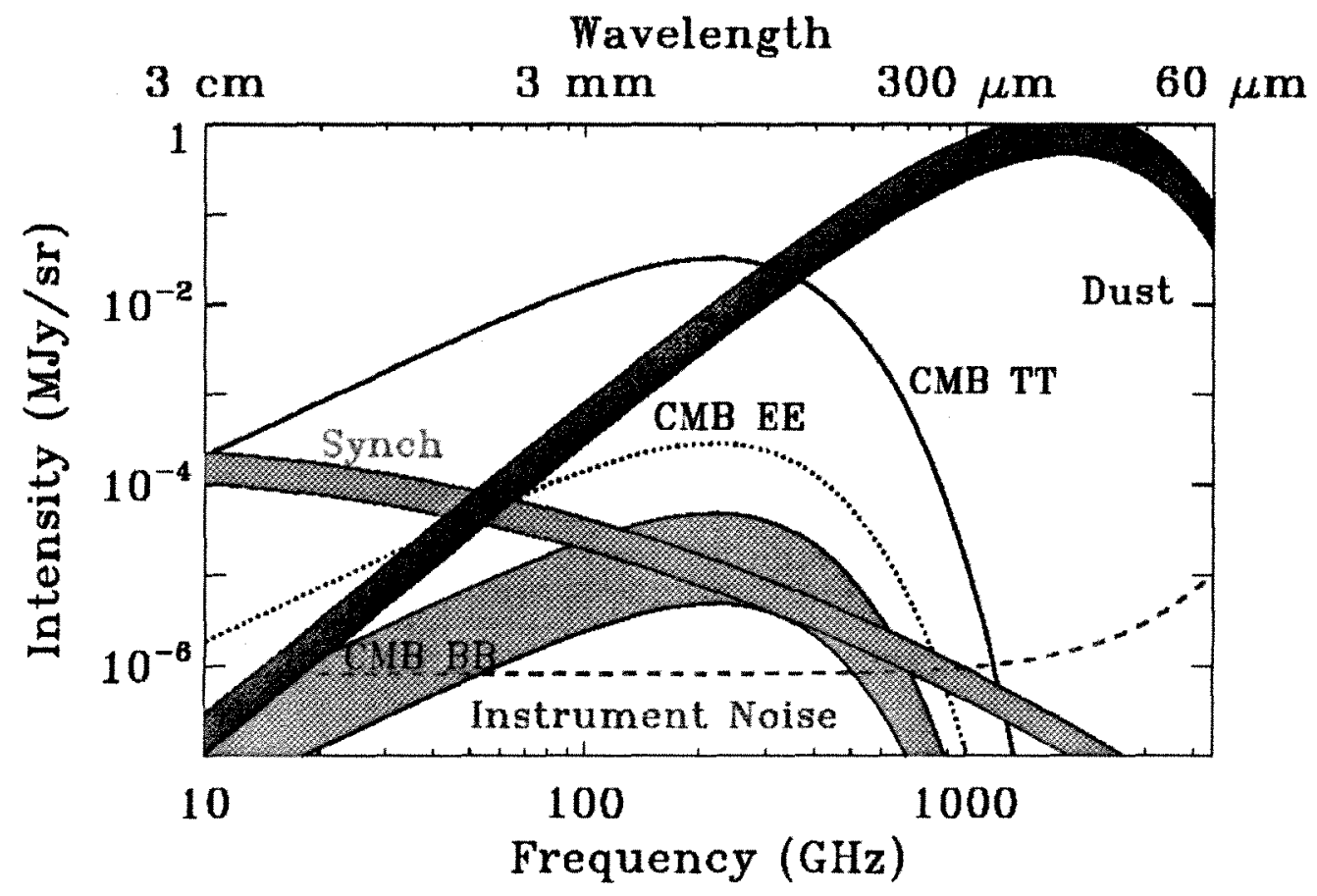

Figure 2. Polarized emission from Galactic synchrotron (green) and dust (blue) is brighter than CMB polarization (grey) at all wavelengths. PIXIE observes over 500 spectral bands from $1 \mathrm{~cm}$ to $50 \mu \mathrm{m}$ wavelength to separate CMB from foreground emission. 

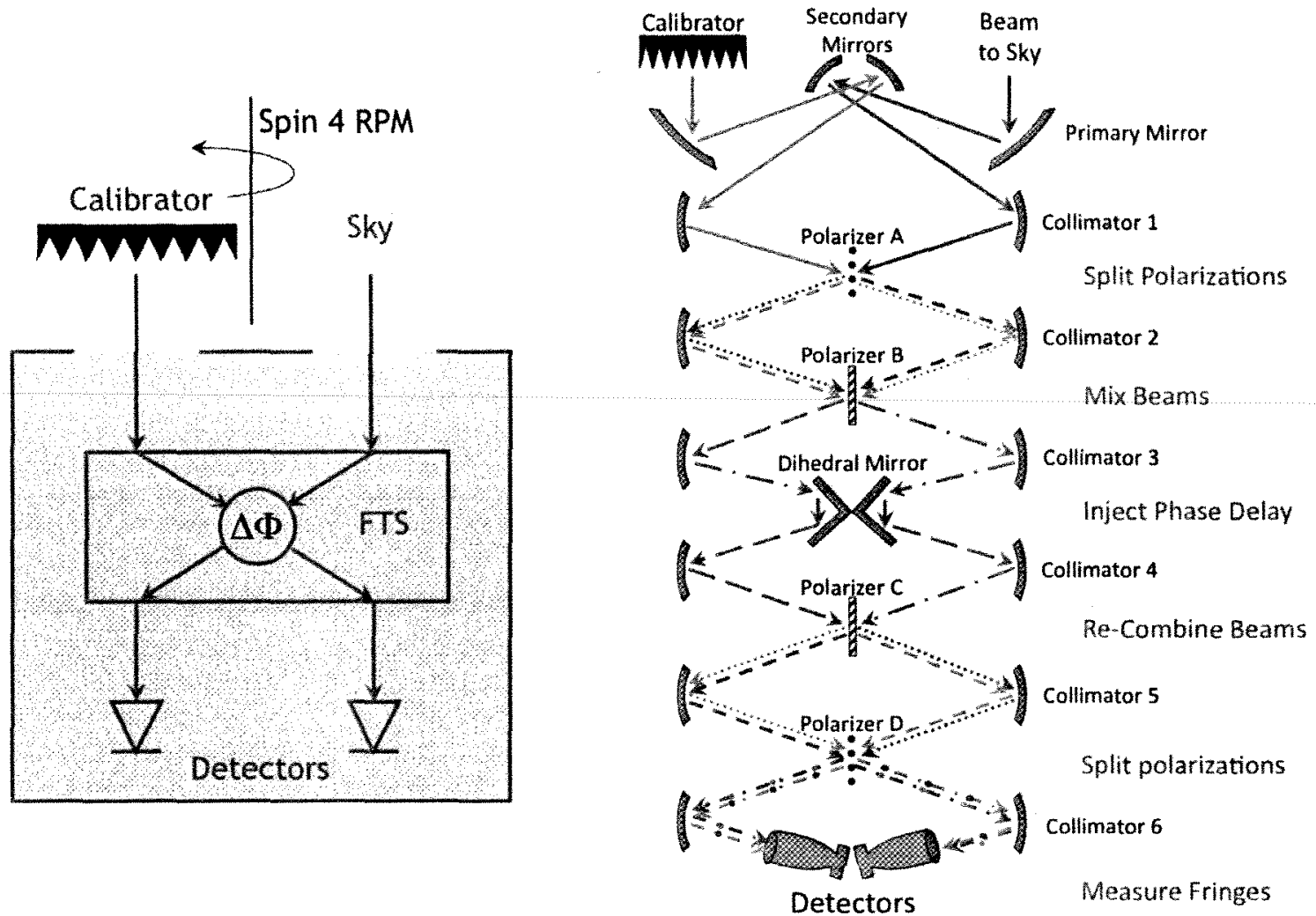

Figure 3. Schematic showing the instrument concept (left) and optical layout (right). The fringe pattern at the detector is the Fourier transform of the frequency spectrum of the Stokes $Q$ linear polarization difference between the two input beams. An unpolarized blackbody calibrator can be inserted to fill either beam to provide absolute spectral information, while the instrument spin modulates the polarization to map the full IQU Stokes parameters on the sky.

Figure 3 shows a schematic of the instrument optical design. Light from 2 independent beams enters a Michelson interferometer. Polarizing wire grids separate the two orthogonal linear polarizations from each input beam, mix the beams using another grid oriented $45^{\circ}$ with respect to the first grid, and then route them to a dihedral mirror assembly which moves to inject an optical phase delay. The phase-delayed beams are then re-combined and re-mixed through another set of grids. As the dihedral mirrors move, a pair of polarization-sensitive bolometers in each of two concentrator feeds measure the resulting fringe pattern from the mixed beams. Let $\vec{E}=E_{x} \hat{x}+E_{y} \hat{y}$ represent the electric field incident from the sky. The power at the detectors as a function of the mirror optical position $z$ may be written

$$
\begin{aligned}
& P_{L x}=\frac{1}{2} \int\left(E_{L x}^{2}+E_{R y}^{2}\right)+\left(E_{L x}^{2}-E_{R y}^{2}\right) \cos (z \omega / c) d \omega \\
& P_{L y}=\frac{1}{2} \int\left(E_{L y}^{2}+E_{R x}^{2}\right)+\left(E_{L y}^{2}-E_{R x}^{2}\right) \cos (z \omega / c) d \omega
\end{aligned}
$$

for the two detectors sharing the left-side feed, where $L$ and $R$ denote the left and right beams on the sky (similar expressions apply for the two detectors sharing the right-side feed). We can express the detected fringe pattern as a constant term (not modulated by the mirror movement) plus a modulated term proportional to the Fourier transform of the difference spectrum between the $\hat{x}$ polarization from one beam and the $\hat{y}$ polarization from the other beam. 
PIXIE operates in a nulled configuration. An unpolarized blackbody calibrator can be moved to fill either the left beam, the right beam, or to leave both beams open to the sky. The spacecraft spins at 4 RPM about the beam axis to repeatedly interchange $\hat{x}$ and $\hat{y}$ on the detectors. The sky signal then becomes

$$
\vec{E}=\left(E_{x} \cos \gamma+E_{y} \sin \gamma\right) \hat{x}^{\prime}+\left(E_{y} \cos \gamma-E_{x} \sin \gamma\right) \hat{y}^{\prime}
$$

in coordinates $\left[\hat{x}^{\prime}, \hat{y}^{\prime}\right]$ fixed with respect to the instrument, where $\gamma$ is the spacecraft rotation angle relating the instrument and sky coordinate systems. Since the mirror stroke at $1 \mathrm{~Hz}$ is fast compared to the $4 \mathrm{RPM}$ spacecraft spin, we may perform a Fourier transform of the fringe pattern each mirror stroke to obtain the difference spectra

$$
S_{v}^{L x}=\frac{1}{4}\left[I_{v}^{L}-I_{v}^{R}+Q_{v} \cos (2 \gamma)+U_{v} \sin (2 \gamma)\right]
$$

for the $\hat{x}$ detector in the left feed, where $I=E_{x}^{2}+E_{y}^{2}, Q=E_{x}^{2}-E_{y}^{2}$, and $U=2 E_{x} E_{y}$ are the Stokes polarization parameters and $S_{v}$ denotes the synthesized frequency spectrum in bins $v$ set by the mirror throw and sampling. PIXIE thus measures the full frequency spectrum of the $I, Q$, and $U$ parameters, referenced to an absolute calibration standard, independently for each pixel in the sky. When the calibrator blocks either beam, the fringe pattern encodes information on both the temperature distribution on the sky (including the monopole) as well as the linear polarization. When both beams view the sky the instrument nulls all unpolarized emission so that the fringe pattern responds only to the sky polarization (Eq.4). Interleaving observations with and without the calibrator allows straightforward transfer of the absolute calibration scale to linear polarization, while providing a valuable cross-check of the polarization solutions obtained in each mode.

Figure 4 shows a possible configuration for the PIXIE observatory. The two parallel beams point along the spacecraft spin axis, which is maintained perpendicular to the Sun line and nearly anti-nadir to minimize signals from the Sun or the Earth. An external blackbody calibrator moves to cover either beam and can be stowed to leave both beams open to the sky. The calibrator, beam-forming optics, beam splitters, and interferometer are maintained at $2.725 \mathrm{~K}$ to remain in thermal equilibrium with the sky. Each semiconductor bolometer is cooled to base temperature $0.1 \mathrm{~K}$ using an adiabatic demagnetization refrigerator. The observatory operates from a polar sun-synchronous orbit to allow an unobstructed view to deep space while avoiding emission from the Sun or Earth.
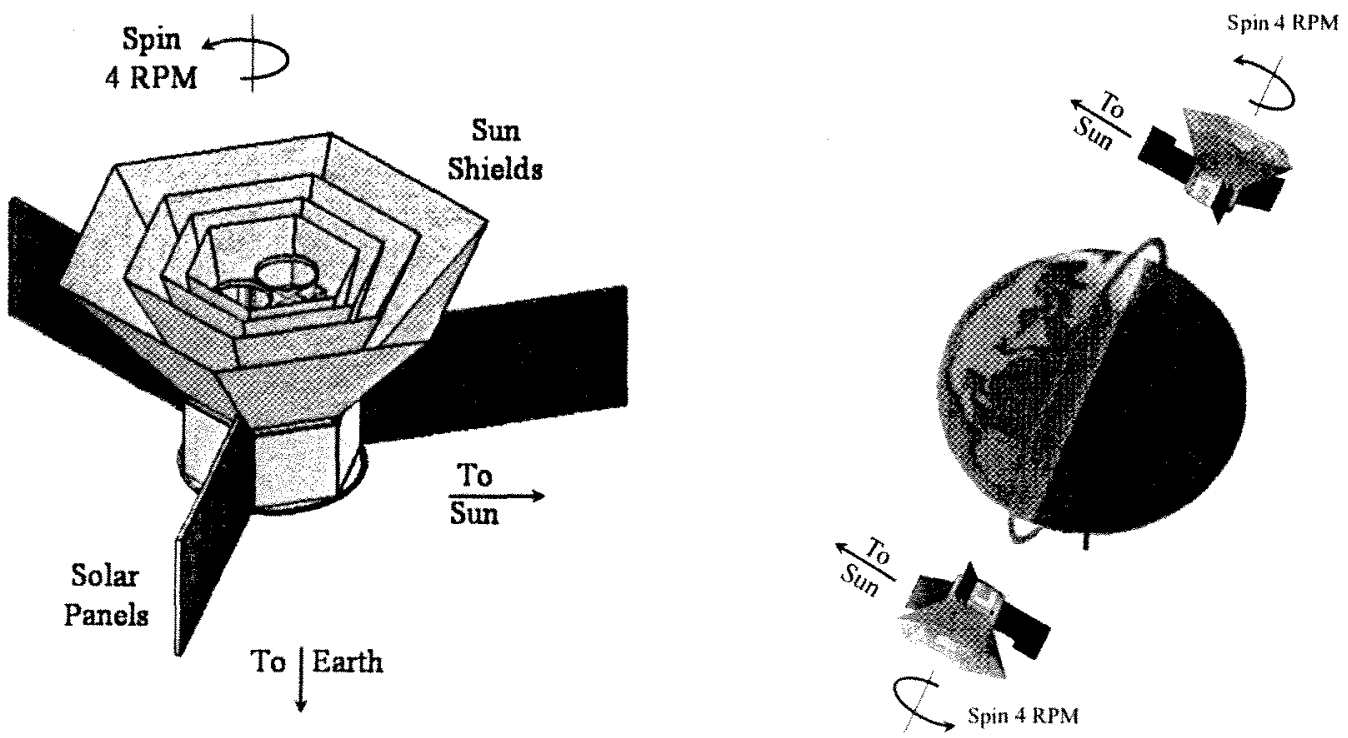

Figure 4. Possible configuration for the PIXIE observatory. The instrument is maintained at $2.7 \mathrm{~K}$ and is surrounded by shields to block radiation from the Sun or Earth. It observes from a polar sun-synchronous orbit. The rapid spin efficiently separates Stokes $I, Q$, and $U$ parameters independently within each pixel to provide a nearly diagonal pixel covariance matrix. 


\section{SENSITIVITY AND SYSTEMATIC ERRORS}

The PIXIE instrument design meets the simultaneous design goals of sensitivity, foreground separation, and systematic error rejection necessary to detect the inflationary signal. The gravity wave signal is generally characterized by the ratio of power in the tensor and scalar modes, $r=T / S$. The simplest inflationary models predict $0.01<r<0$. 16, corresponding to B-mode polarization amplitude $30-100 \mathrm{nK}^{7,8,9}$. Current state-of-the-art instruments ${ }^{10,11,12}$ search for the inflationary gravity wave signal using kilo-pixel arrays of transition-edge superconducting bolometers. The push to kilo-pixel detector arrays results from the need to overcome photon noise statistics. Strictly speaking, however, the need is for more photons, not necessarily more detectors. The spectral density of photon noise is given by ${ }^{13}$

$$
N E P_{\text {phoon }}^{2}=\frac{4 A \Omega(k T)^{5}}{c^{2}} \int \alpha \varepsilon f \frac{x^{4}}{h^{3}-1}\left(1+\frac{\alpha \varepsilon f}{e^{x}-1}\right) d x
$$

where $x=h v / k T, v$ is the observing frequency, A is the detector area, $\Omega$ is the detector solid angle, $\alpha$ is the detector absorptivity, $T$ is the physical temperature of the source, $\varepsilon$ is the source emissivity, and $f$ is the power transmission through the optics. The light-gathering ability of an instrument is specified by its etendu $A \Omega$. For diffraction-limited single-mode optics, the etendu and wavelength are related as $A \Omega=\lambda^{2}$ so that the beam size scales with the observing wavelength. For multi-moded optics, however, the beam size is fixed and the number of modes $\mathrm{N}$ scales as $\mathrm{N}=A \Omega / \lambda^{2}$. PIXIE has 4 detectors each with etendu $4 \mathrm{~cm}^{2}$ sr. The spectrometer produces spectra with bin width $15 \mathrm{GHz}$. Over just the frequency range $30--600 \mathrm{GHz}$ where the CMB is brightest, PIXIE observes over 44,000 independent modes in 40 spectral bins, compared to $\sim 1500$ single-mode detectors in $\sim 6$ bins for comparable CMB polarimeters. The full spectrum covers the range $30 \mathrm{GHz}$ to $6 \mathrm{THz}(1 \mathrm{~cm}$ to $50 \mu \mathrm{m})$ to provide high signal-to-noise polarization maps of all relevant Galactic foregrounds.

The frequency bins in the synthesized spectra are set by the mirror throw and detector sampling. As the mirror moves, we obtain $N_{\mathrm{s}}$ detector samples over an optical path length $\pm \Delta z$. The Fourier transform of the sampled fringe

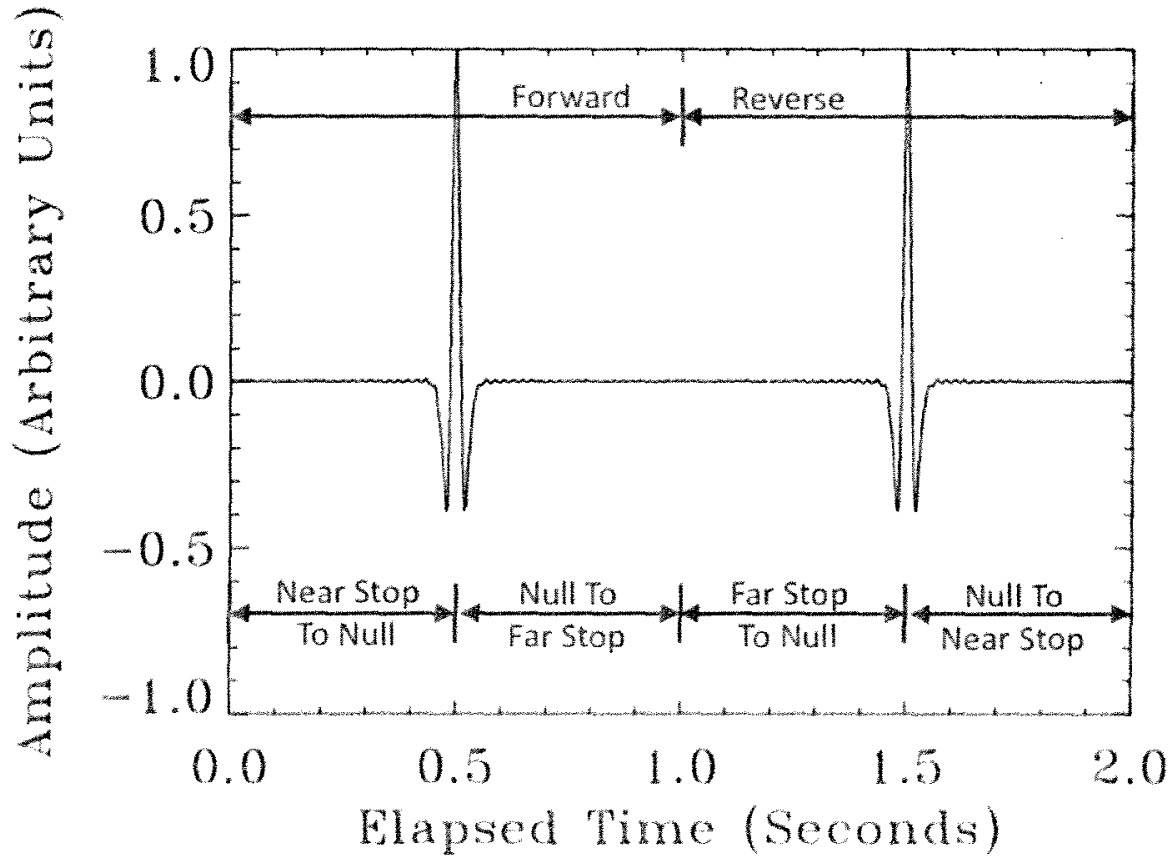

Figure 5. Simulation showing the observed fringe pattern on a single detector as the mirror sweeps back and forth through 2 complete interferograms while observing an unpolarized CMB signal. Sky signals must obey multiple timeand space-reversal symmetries in the time-ordered data, allowing straightforward identification and removal of instrumental signals. 
pattern returns frequencies $n \times c /(2 \Delta z)$ where $n=0,1,2, \ldots N_{\mathrm{s}} / 2$. With $N_{\mathrm{s}}=1024$ and $\Delta \mathrm{L}=1 \mathrm{~cm}$, we obtain 512 bins of width $15 \mathrm{GHz}$ each. The corresponding physical movement $x=z /[4 \cos (\alpha) \cos (\delta / 2)]=2.6 \mathrm{~mm}$ accounts for the folded optics as well as the off-axis optical path $\left(\alpha=15^{\circ}\right)$ and beam divergence $\left(\delta=7^{\circ}\right)$. A full cycle of the mirror from one endpoint to the other and back thus contains 2 complete interferograms (Figure 5).

A Fourier transform uniformly sampled from -1 to +1 has sharp edges at \pm 1 , leading to ringing in the frequency domain. In addition, the $\mathrm{CMB}$ only produces fringes near zero path length -- observations at longer optical path, necessary to provide sufficiently narrow frequency bins, have almost no CMB signal and thus contribute little to the CMB sensitivity. We minimize ringing and maximize sensitivity by varying the mirror stroke to apodize the Fourier transform. A commonly used apodization is $\left(1-z^{2}\right)^{2}$. PIXIE observes any given location on the sky for at least 13 consecutive orbits. Each orbit uses a different mirror stroke length, ranging from a shortest stroke $\Delta z=3.3 \mathrm{~mm}$ (physical movement $\pm 0.9 \mathrm{~mm}$ ) to a longest stroke $10 \mathrm{~mm}$ (physical movement $\pm 2.6 \mathrm{~mm}$ ). Figure 8 shows the resulting apodization. By spending relatively more time near zero delay, we apodize the Fourier transform to retain narrow frequency bins while simultaneously increasing the sensitivity to CMB signals by $37 \%$ compared to uniform sampling.

The PIXIE observing strategy provides immunity to $1 /$ f noise or other instrumental drifts. A single interferogram takes data as the mirror moves from one endpoint through the null to the other endpoint. Not only does this provide null tests against systematic errors, but the spatial symmetry about zero path length forces the sky signal entirely into the real part of the Fourier transform. The imaginary part corresponds to the anti-symmetric component of the sampled data and will consist only of instrument noise and systematic errors. This provides a powerful check on systematics while yielding a clean measurement of the instrument noise (including any $1 / \mathrm{f}$ component) fully coincident with the noise in the final sky spectra. In addition, the Fourier transform operates on short stretches of data, ranging from $330 \mathrm{~ms}$ to 1 second (depending on the mirror apodization stroke). Each interferogram is independent, so 1/f noise or drifts on time scales much longer than 1 second will appear as a constant slope or a low-order polynomial in any given fringe pattern. Such artifacts affect only the lowest few frequency bins of the Fourier transform and do not project onto either the $\mathrm{CMB}$ or foreground spectra.

\subsection{Amplitude Modulation}

The spacecraft spin provides additional protection against systematic errors. The fringe pattern shown in Figure 5 corresponds to an unpolarized CMB signal. The 4 RPM rotation introduces an additional modulation for polarized emission from the sky. Figure 7 shows the resulting fringe pattern. Unlike simple polarization-sensitive bolometers,

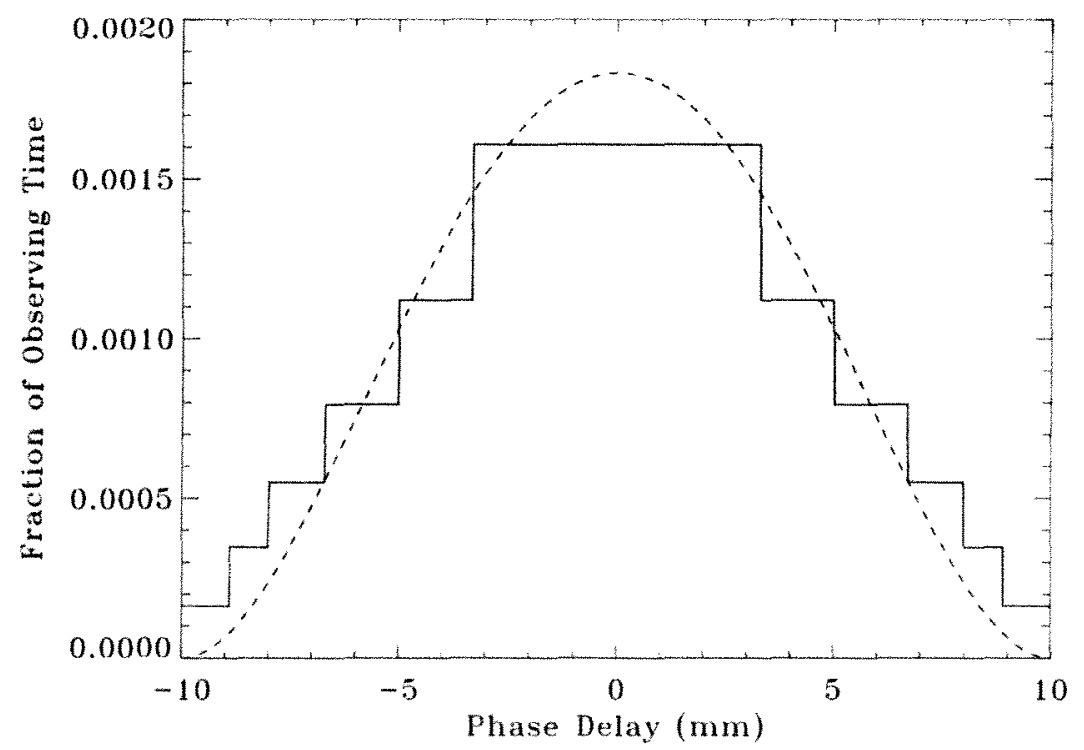

Figure 6. Apodization of the optical phase delay created by scanning the mirror through different path lengths on different orbits. The actual apodization (solid line) closely approximates the optimum (dashed line) and suppresses ringing while yielding a $37 \%$ increase in sensitivity to the $\mathrm{CMB}$ compared to uniform sampling. 


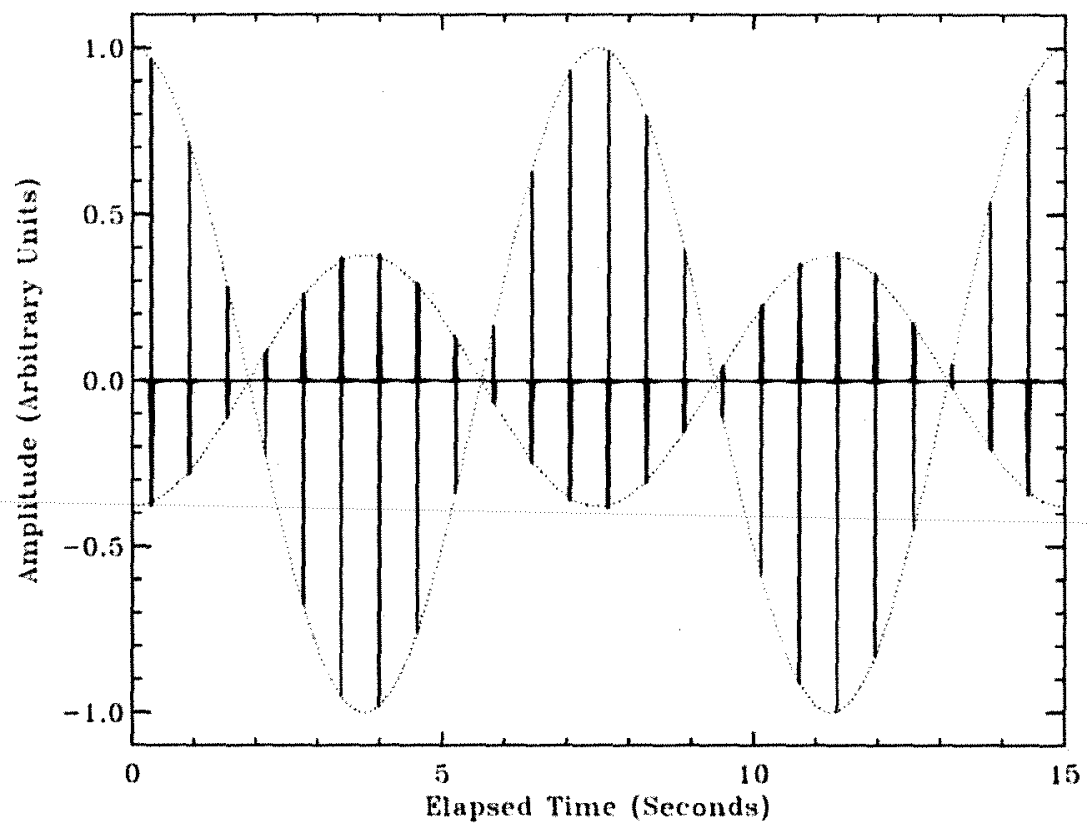

Figure 7. Simulated fringe patterns from a single detector observing polarized CMB. The instrument rotation imposes an amplitude modulation (dotted envelope) on the entire fringe pattern, efficiently suppressing spurious signals locked at a single frequency.

where instrument rotation produces a sinusoidal response to polarized sky signals, PIXIE's rotation produces amplitude modulation of the entire fringe pattern. The resulting modulated fringe pattern is readily distinguished from a spinlocked sine wave, efficiently suppressing any spin-synchronous drifts.

The amplitude-modulated interference fringes are a powerful guard against broad classes of systematic effects. Consider, for instance, an additive signal of the form $V_{\text {det }}=V_{o}(t)+V_{s}(t)$, where $V_{o}(t)$ is the detector output from the optical fringe pattern of a true sky signal and $V_{s}(t)=\sin (\omega t+\phi)$ is a sine-wave systematic error (e.g. from the back-end electronics). Even if the systematic error signal occurs at a particularly important frequency related to the mirror stroke or spacecraft spin, its overlap with the amplitude-modulated CMB fringe pattern is small. We quantify the susceptibility to additive systematic error signals by simulating the PIXIE time-ordered data with the period of the additive term corresponding to either the orbit period, spin period, half the spin period, or the mirror stroke period. We then Fourier transform the simulated data from each mirror stroke (Eq. 2) to obtain sky spectra, tag each spectrum with the sky coordinates and associated spin angle $\gamma$, and solve for the Stokes $l, Q$, and $U$ parameters. We then compare the computed spectra $Q_{v}(E q 4)$ to a differential Planck spectrum to quantify the projection of systematic error signals onto the CMB polarization. Since the sinusoidal error signal lacks power on short time scales (short spatial frequencies in the interferogram), the power in the Fourier-transformed spectra is concentrated in the first few frequency bins. The projection onto $\mathrm{CMB}$ polarization is small, with rejection better than $-65 \mathrm{~dB}$.

\subsection{Sum and Difference Spectra}

PIXIE's symmetric design reduces sensitivity to other potential systematic errors. Consider the effects of non-ideal optical systems. The rotation of a non-circular (elliptical) beam across an un-polarized sky will produce a spurious polarization signal (at twice the spin frequency) wherever the un-polarized sky contains a local saddle point. The aliasing of power from a local temperature quadrupole moment to a false polarization signal grows rapidly with beam ellipticity. PLXIE has 4 polarization-sensitive bolometers: one pair in orthogonal linear polarizations behind one feed, and a redundant pair behind a second feed. If we consider the output of a single detector, we find significant coupling to beam ellipticity: requiring the systematic error signal to remain below a few nK requires the beam ellipticity $e^{<3 \times 10^{-4}}$ [14]. Comparing data between bolometers significantly relaxes the dependence on beam ellipticity. The two detectors in 
each feed share the full optical chain to the sky, differing only in polarization orientation. Their sum and difference spectra (Eq. 4) may be written as

$$
\begin{aligned}
& S_{v}^{\text {sum }}=\frac{1}{2}\left[I_{v}^{L}-I_{v}^{R}+\frac{\delta G}{G}\left(Q_{v} \cos (2 \gamma)+U_{v} \sin (2 \gamma)\right)\right] \\
& S_{v}^{\text {diff }}=\frac{1}{2}\left[Q_{v} \cos (2 \gamma)+U_{v} \sin (2 \gamma)+\frac{\delta G}{G}\left(I_{v}^{L}-I_{v}^{R}\right)\right]
\end{aligned}
$$

where $\delta G / G$ is the fractional calibration error between the two detectors. The sum is dominated by the unpolarized spectrum, while the difference has contributions from both the polarized sky spectra and a term proportional to the gain error times the unpolarized spectrum. We may then recover the sky spectra $\mathrm{I}, \mathrm{Q}$, and $\mathrm{U}$ using the following algorithm. We generate the detector sum spectra and bin them by sky position to find the unpolarized spectrum $I_{\text {sum }}$ in each pixel, ignoring the second-order term $\delta G / G(Q+U)$. We then generate the detector difference spectra and use the sky position and rotation angle to solve for the polarization spectra $Q$ and $U$ plus an unpolarized correction term $I_{\text {diff. }}$ The final sky solution uses $I=I_{\text {sum }}+I_{\text {diff }}$ to recover the unpolarized gain mismatch term.

The use of sum and difference spectra greatly reduces the $\Delta \mathrm{T} \rightarrow \mathrm{B}$ coupling. This translates into relaxed requirements for instrument beam ellipticity $e$. Simulations show that for calibration error as large as $1 \%$, achieving systematic errors below $3 \mathrm{nK}$ only requires beam ellipticity $e<0.06$. In practice, the calibration error will be much smaller, reducing beam artifacts to negligible levels.

\subsection{Symmetry and Null Tests}

Null tests are an important demonstration that systematic errors are small compared to the instrument noise. PIXIE's highly symmetric design provides a large number of such tests. A few of the more important null tests include:

- Interferograms from a single detector should remain unchanged as the mirror moves forward or backward. Any difference traces errors in the detector response function.

- Interferograms on the same detector taken $1 / 4$ spin apart should have polarized signal opposite in sign. Any difference indicates a residual instrumental polarization.

- Interferograms on the same detector taken $1 / 2$ spin apart should be identical. Any difference indicates cross-polar leakage.

- Interferograms on the left detector should be identical to those from the right detector $1 / 4$ spin later. Any difference indicates cross-polar leakage or detector calibration error between the two sides of the instrument.

- Interferograms taken with the calibrator over one aperture should be opposite to interferograms taken with the calibrator over the other aperture. Any difference indicates asymmetries in the instrument optical path.

- Spectra from a given pixel should be identical when the pixel is revisited. Re-visits occur on all time scales from a single orbit for pixels near the celestial poles to 6 months for pixels near the celestial equator.

In addition, the interferograms are symmetric about zero phase delay so that the sky signal contributes only to the real part of the synthesized spectra. The imaginary part consists of an independent realization of the instrument noise (plus any systematics), obtained simultaneously with the noise realization in the sky signal. All null tests in the list above thus use the imaginary part of the synthesized spectra to characterize the statistical properties of the noise in that test -- PIXIE does not rely on difference tests to determine the noise properties.

\section{FOREGROUNDS AND FREQUENCY SPECTRA}

Four foreground components are relevant for CMB polarization. ${ }^{15,16}$ Synchrotron emission results from cosmic ray electrons accelerated in Galactic magnetic fields; the amplitude (in intensity units) scales as $S_{\text {synch }} \sim v^{\alpha 8}$ where the spectral index $\alpha_{3}-1$ is spatially variable and steepens with increasing frequency. Polarized dust emission results from 
grains aligned in the same magnetic field and scales as $S_{\text {dust }} \sim v^{\text {ad }} B_{v}\left(T_{\text {dust }}\right)$ where $\alpha_{\mathrm{d}} \sim 2$ and $B_{v}$ is a blackbody at temperature $T_{\text {dust. }}$. Free-free emission from electron-ion collisions in the warm interstellar medium is largely unpolarized, but may acquire a small polarized component in scattering from ubiquituous dust grains. In addition, electric dipole emission from a population of small, rapidly spinning dust grains dominates Galactic emission at wavelengths near $1 \mathrm{~cm} .^{17.18}$ Although the intrinsic polarization from such emission is predicted to be less than a few percent ${ }^{19}$, it complicates foreground cleaning through confusion with the synchrotron component of diffuse continuum radio emission.

These emission mechanisms are smooth functions of frequency so that multi-frequency observations can be combined to model and remove foreground contamination. The required number of frequency channels depends on the number of fitted parameters. The most optimistic technique fixes the spectral dependence and fits only the CMB, synchrotron, and dust amplitudes, requiring a minimum of 3 frequency channels. Fitting additional parameters (synchrotron spectral index, synchrotron spectral curvature, free-free or spinning dust contribution, dust color temperature, dust spectral index, dust alignment) requires additional frequency channels.

PIXIE samples the observed fringe pattern at 1024 spatial frequencies to synthesize frequency spectra in 512 bins spaced $15 \mathrm{GHz}$ apart. A low-pass filter on the front-end optics blocks zodiacal light, but no other passband filters are required. The broad spectral coverage and clean optical path provide an ideal data set to constrain models of foreground emission. The number of frequency channels is much larger than any plausible number of foreground parameters, allowing straightforward statistical assessment of the utility of adding additional foreground parameters. Foregrounds are observed through the same optics and using the same calibration as the CMB, automatically including frequency bands where the foregrounds dominate the sky as well as bands where the CMB is more prominent. The large number of frequency bands provides a minimum "noise penalty" for linear combinations of frequency channels used to reject foreground emission. Assuming (for illustration) a simple model with power-law synchrotron and dust foregrounds ( $\alpha_{\mathrm{s}}$ and $\alpha_{d}$ independent of either sky position or frequency), the noise in a foreground-cleaned PIXIE map is only $8 \%$ worse than the ideal case of noise weighting with no foregrounds.

The broad frequency range of the PIXIE data provides rich ancillary science in addition to the primary goal of inflationary science. Thomson scattering of $\mathrm{CMB}$ photons from free electrons at the epoch of reionization sources E-mode polarization on large angular scales. The same scattering necessarily distorts the unpolarized CMB away from a blackbody. PIXIE will observe both the E-mode polarization and unpolarized spectral distortion. The combination can be used to fix not only the optical depth, but also the gas temperature, and hence provide information on the ionization mechanism at redshift $z \sim 10$. PIXIE data in the far-infrared will characterize the monopole, dipole, and higher-order power spectrum of the far-IR background to test the matter distribution at redshift $z \sim 3$. PIXIE will measure line emission from the interstellar medium, including the prominent $\mathrm{CII}(158 \mu \mathrm{m})$, NII $(205 \mu \mathrm{m})$, and OI $(63 \mu \mathrm{m})$ lines as well as the CO series.

\section{DETECTOR IMPLEMENTATION}

PIXIE requires detectors with large absorbing area (etendu $4 \mathrm{~cm}^{2} \mathrm{sr}$ ) capable of detecting a single linear polarization (cross polar response $1 \%$ or smaller). The longest mirror stroke samples the detector 1024 times per 1 second stroke, so the detector time constant should be of order $1 \mathrm{~ms}$. To minimize lost time from cosmic-ray hits, the absorbing area for photons should be large compared to the geometric cross section. Finally, ultra-broad-band detection at the photon limit requires device (phonon) $\mathrm{NEP}<10^{-16} \mathrm{~W} \mathrm{~Hz}^{-1 / 2}$.

The PIXIE detectors require absorber area a factor of ten larger than the detectors flown on the BOOMERANG suborbital instrument or ESA's Planck satellite. ${ }^{20,21}$ Existing polarization-sensitive bolometer designs can not simply be scaled to meet the PIXIE requirements. As the detector linear scale becomes larger than a wavelength, thermalization of the active absorber area becomes problematic. This problem is especially acute for multi-moded optics where significant spatial gradients in optical power can occur across the absorber. As the instrument beam scans across the sky, these gradients will shift across the absorber. The response of the thermistor will be a complex sum of the thermal response along individual strands, with power absorbed far from the thermistor delayed in time with respect to power absorbed 


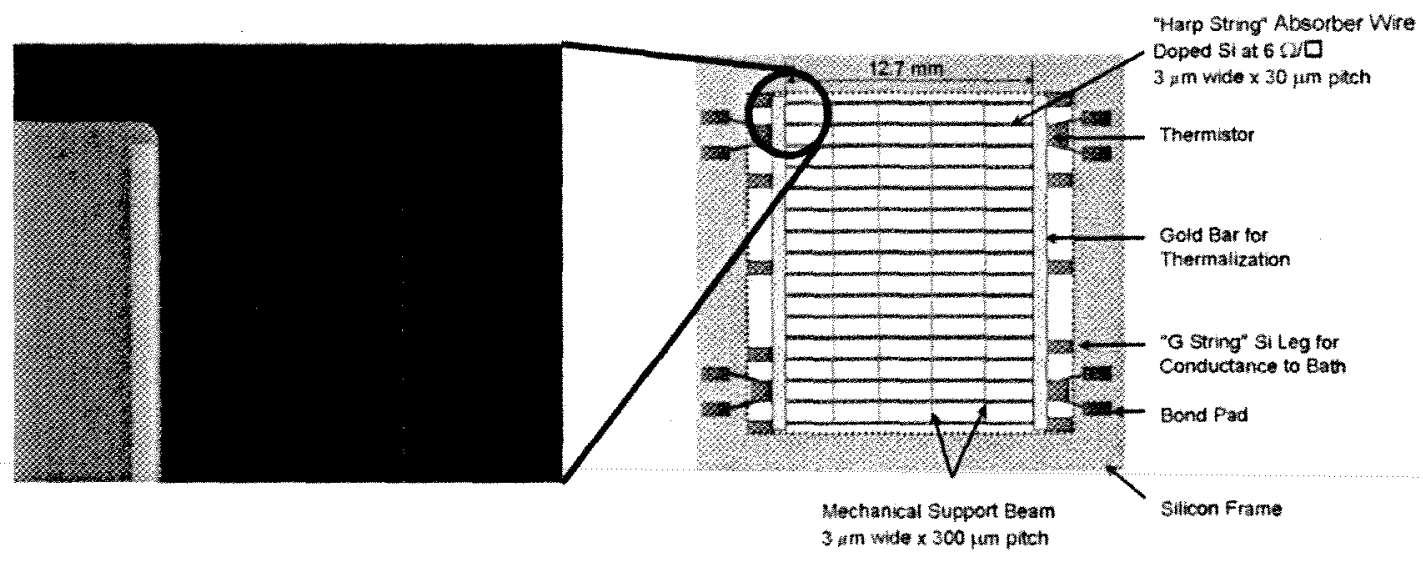

Figure 8. Schematic of PIXIE polarization-sensitive bolometer. Thin wires of doped crystalline silicon absorb a single linear polarization. Gold bars depositied over the ends of the wires thermalize the device. The measured time constant is $0.6 \mathrm{~ms}$. Four ion-implanted thermistors provide temperature readout. The inset at left shows the absorbing strands and thermalizing bar on the fabricated device

nearby. The time-ordered signal from the thermistor will then include a contribution from sources other than the interferometer fringes (Eq. 2), aliasing power from bright unpolarized sources into the much fainter polarization.

We have developed a large-area polarization-sensitive bolometer for multi-moded optical systems such as PIXIE. Figure 8 shows a schematic of the PIXIE detector. It consists of a set of thin, parallel strands of doped crystalline silicon, micro-machined from an ion-implanted layer and suspended from an un-doped crystalline silicon frame. PIXIE will operate from $1 \mathrm{~cm}$ to $50 \mu \mathrm{m}$ wavelength; the wire pitch is thus set at $30 \mu \mathrm{m}$. Un-doped cross beams spaced every 300 $\mu \mathrm{m}$ provide additional mechanical support without adding significantly to the geometric cross section; the geometric filling fraction is only $11 \%$. We deviate from the PSB design in two significant ways. The entire device, including the absorbing strands and thermal link to the bath, is made from crystalline silicon to increase the thermal conductance and decrease the absorber time constant. To stabilize the thermal response of the entire detector, we add two thick $(0.5 \mu \mathrm{m})$ gold bars along the edges where the absorbing strings connect to the frame. The gold bars dominate the heat capacity of the detector to allow thermalization of the contribution from individual wires. A set of 4 ion-implanted silicon thermistors are thermally anchored to the gold bars to monitor the temperature. The time constant of the device is set by the thermal conductance $G$ of a series of silicon legs linking the absorber/bar composite to the silicon frame, which also serve to mechanically support and stiffen the absorbing structure. The detector is physically large, with detector area $12.7 \times 12.7 \mathrm{~mm}$. Despite the large area, the time constant for heat transport across the absorbing strands is short ( $\tau=0.6 \pm 0.1 \mathrm{~ms}$ ), validating the choice of crystalline silicon for the detector substrate.

\section{REFERENCES}

[1] Kamionkowski, M., Kosowsky, A., and Stebbins, A., "Statistics of cosmic microwave background polarization," PRD, 55, 7368 (1997).

[2] Seljak, U, and Zaldarriaga, M., "Signature of Gravity Waves in the Polarization of the Microwave Background", PRL, 78, 2054 (1997).

[3] Hu, W., and White, M.J., "A CMB polarization primer," New Astronomy, 2, 323 (1997).

[4] Lyth, D.H., "What Would We Learn By Detecting a Gravitational Wave Signal in the Cosmic Microwave Background", PRL, 78, 1861 (1997).

[5] Bock, J., et al., "Task Force on Cosmic Microwave Background Research", arXiv:astro-ph/0604101v1 (2006).

[6] Dodelson, S., et al., "the Origin of the Universe as Revealed Through the Polarization of the Cosmic Microwave Background", Astro2010 White Paper 67, arXiv:0902.3796 (2009). 
[7] Spergel, D.N., et al., "First-Year Wilkinson Microwave Anisotropy Probe (WMAP) Observations: Determination of Cosmological Parameters," ApJS, 148, 175 (2007).

[8] Linde, A.D., "Current Understanding of Inflation," New Astronomy Reviews, 49, 35 (2005).

[9] Lyth, D.H., and Riotto, A., "Particle Physics Models of Inflation and the Cosmological Density Perturbation," Phys. Rep., 314, 1 (1999).

[10] Montroy, T.E., et al., " SPIDER: a new balloon-borne experiment to measure CMB polarization on large angular scales," Proc SPIE, 6267, 6267R (2006).

[11] Oxley, P., et al., "The EBEX Experiment", Proc SPIE, 5543, 320 (2004).

[12] Chuss, D., et al. "Primordial Inflation Polarization Explorer". Proc. SPIE, 7741 (2010)

[13] Mather, J.C., "Bolometer noise: nonequilibrium theory," Applied Optics, 21, 1125 (1982).

[14] Shimon, M., "CMB polarization systematics due to beam asymmetry: Impact on inflationary science", PRD, 77, 083003 (2008).

[15] Page, L, et al., "Three-Year Wilkinson Microwave Anisotropy Probe (WMAP) Observations: Polarization Analysis", ApJS, 170, 335 (2007).

[16] Gold, B, et al., "Five-Year Wilkinson Microwave Anisotropy Probe (WMAP) Observations: Galactic Foreground Emission", ApJS, 180, 265 (2009).

[17] Kogut, A., et al., "ARCADE 2 Observations of Galactic Radio Emission", ApJ, submitted, arXiv:0901.0562 (2009).

[18] Gold, B., et al., "Seven-Year Wilkinson Microwave Anisotropy Probe (WMAP) Observations: Galactic Foreground Emission", ApJS, in press (2010).

[19] Lazarian, A., and Draine, B. T., "Resonance Paramagnetic Relaxation and Alignment of Small Grains", ApJL, 536, $15(2000)$.

[20] Jones, W.C., et al, "Instrumental and analytic methods for bolometric polarimetry," A\&A, 470, 771 (2007).

[21] Holmes, W.A., et al., "Initial Test Results on Bolometers for the Planck High Frequency Instrument," Applied Optics, 47, 5996 (2008).

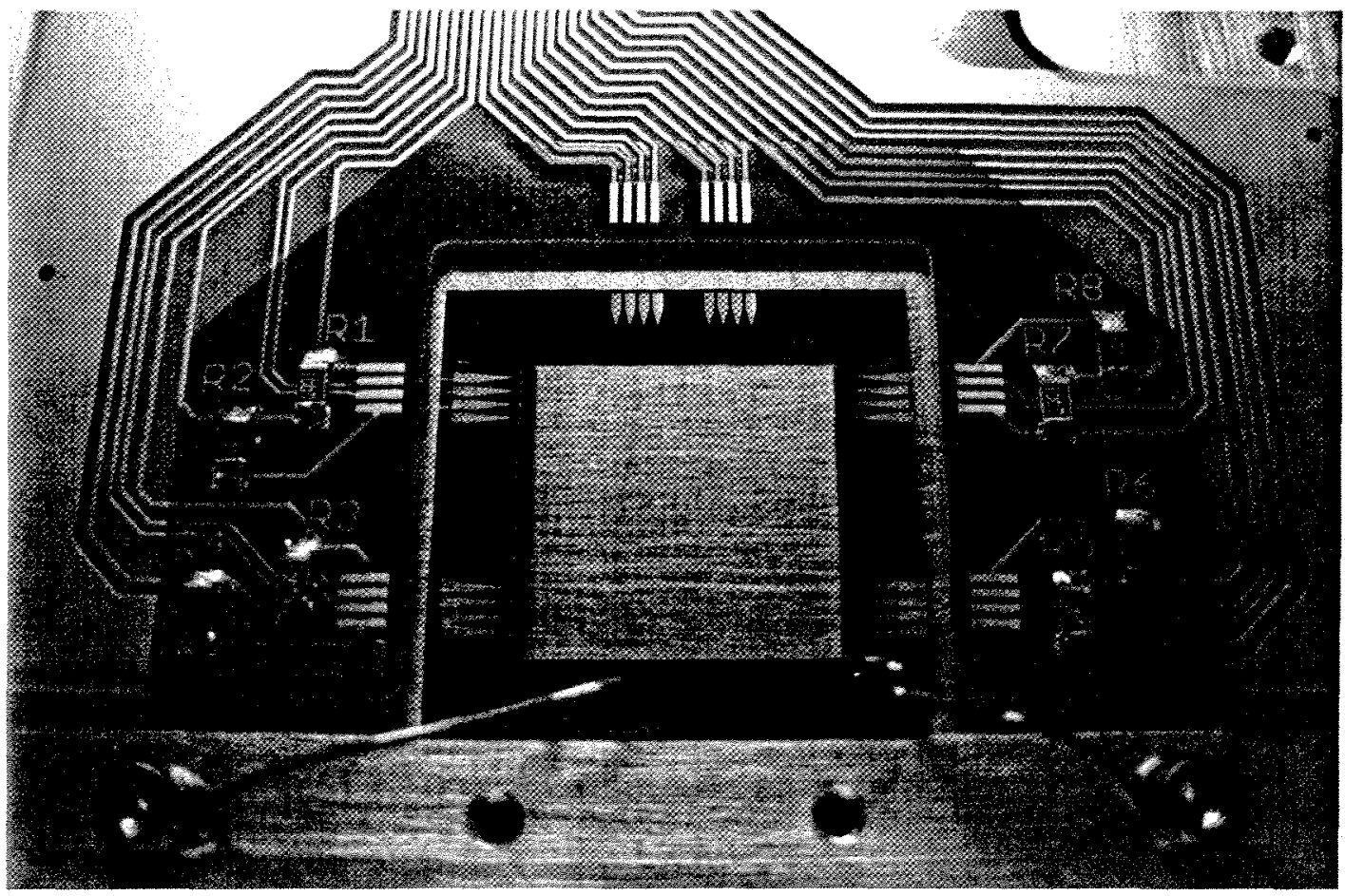

Figure 9: Polarization-sensitive bolometer during thermal testing. 